\title{
Ghrelin protects adult rat hippocampal neural stem cells from excessive autophagy during oxygen-glucose deprivation
}

\author{
Hyunju Chung 1 ) , Junghyun Choi $\left.{ }^{2}\right) *$ and Seungjoon Park ${ }^{3)}$ \\ 1) Department of Core Research Laboratory, Clinical Research Institute, Kyung Hee University Hospital at Gangdong, Seoul, Korea \\ 2) Department of Biomedical Science, Graduate School, Kyung Hee University, Seoul, Korea \\ 3) Department of Pharmacology and Medical Research Center for Bioreaction to ROS and Biomedical Science Institute, School of \\ Medicine, Kyung Hee University, Seoul, Korea
}

\begin{abstract}
Ghrelin functions as a neuroprotective agent and saves neurons from various insults include ischemic injury. However, it remains to be elucidated whether ghrelin protects neuronal cells against ischemic injury-induced excessive autophagy. Autophagy is required for the maintenance of neural stem cell homeostasis. However, regarding autophagic cell death, it is commonly assumed that excessive autophagy leads to self-elimination of mammalian cells. The purpose of this study was to investigate the potential neuroprotection effects of ghrelin from excessive autophagy in adult rat hippocampal neural stem cells (NSCs). Oxygen-Glucose Deprivation (OGD) strongly induces autophagy in adult rat hippocampal NSCs. Ghrelin treatment inhibited OGD-induced cell death of adult rat hippocampal NSCs assessed by cell-counting-kit- 8 assay. Ghrelin also suppressed OGD-induced excessive autophagy activity. The protective effect of ghrelin was accompanied by an increased expression levels of Bcl-2, p-62 and decreased expression level of LC3-II, Beclin-1 by Western blot. Furthermore, ghrelin reduced autophagosome formation and number of GFP-LC3 transfected puncta. In conclusion, our data suggest that ghrelin protects adult rat hippocampal NSCs from excessive autophagy in experimental stroke (oxygen-glucose deprivation) model. Regulating autophagic activity may be a potential optimizing target for promoting adult rat hippocampal NSCs based therapy for stroke.
\end{abstract}

Key words: Ghrelin, Excessive autophagy, Oxygen-glucose deprivation, Neuroprotection, Hippocampus

GHRELIN is a 28-amino acid peptide hormone type of growth hormone $(\mathrm{GH})$ secretagogues (GHS) was first isolated from stomach in 1999 by Kojima [1]. Ghrelin with n-octanoyl modification at Ser3 act through the GHS-receptor 1a (GHS-R) which is a G protein-coupled receptor and stimulates the release of GH [2]. Ghrelin also has physiological actions throughout the body, including effects on exocrine and [3], carbohydrate metabolism [4]. Moreover, ghrein shows nonendocrine

Submitted Aug. 1, 2017; Accepted Sep. 6, 2017 as EJ17-0281

Released online in J-STAGE as advance publication Oct. 21, 2017

Correspondence to: Seungjoon Park, M.D., Ph.D., Department of Pharmacology and Medical Research Center for Bioreaction to ROS and Biomedical Science Institute, School of Medicine, Kyung Hee University, 26, Kyungheedae-ro, Dongdaemun-gu, Seoul, 02447, Korea

E-mail: sjpark@khu.ac.kr

*These authors contributed equally to this work. effects to control differ brain functions, such as protection of neuronal cells [5-8]. In addition, ghrelin improves functional recovery after traumatic by inhibition of apoptotic cell death pathway [9]. The neuroprotective effect of ghrelin be associated with its inhibition of inflammatory activity [10], oxidative stress [5]. However, the exact protective mechanisms of ghrelin in neuroprotection remain to be further clarified.

Autophagy is the cellular process of self-digestion, acting to remove targeted proteins and organelles under various cellular stresses and/or extrinsic stimuli. In fact, dysregulated autophagy has been involved in many human disease states. Also as a bulk cellular degradation pathway, autophagy has been widely reported in its neuroprotective potential [11-13] and autophagy is thought to be a protective mechanism that sustain cell survival under stress conditions. By contrast, altercation exist whether excessive autophagy activity contribute to 
autophagy induced cell death [14-16], when autophagy is excessively induced, it can result in autophagic cell death, type II programmed cell death (PCD). The majority of the cell death due to cerebral ischemia has been attributed to apoptosis; type I PCD [17]. However there is increasing evidence that excessive autophagy is involved in mediating neuronal cell death during cerebral ischemia $[18,19]$.

In the present study, in order to study the role of ghrelin in hypoxia/ischemia-induced injury, we investigated the effect of ghrelin on the survival of adult rat hippocampal NSCs exposed to hypoxia. Our results suggest that the neuroprotective properties of ghrelin during hypoxic injury are associated with the inhibition of LC3-II, Beclin-1 or activation of Bcl-2, p-62 through the suppression of excessive autophagosome formation. Moreover, our data also suggest that regulation of autophagy activity is associated with the neuroprotective effect of ghrelin.

\section{Materials and Methods}

\section{Materials}

Rat ghrelin was obtained from Peptides International (Louisville, KT, USA). D-Lys-3-GHRP-6 was purchased from Bachem (Torrance, CA, USA). NSC expansion media, DMEM/F12, and B27 supplement were obtained from Gibco/Invitrogen. B-27 is an optimized serum substitute developed for low-density plating and long-term viability and growth of central nervous system (CNS) neurons (Brewer et al. 1993). All tissue culture reagents were obtained from Gibco/Invitrogen, and all other reagents were obtained from Sigma unless otherwise indicated.

\section{Adult rat hippocampal NSCs cultures and treatments}

Adult rat hippocampal NSCs were obtained from Chemicon (Catalog No. SCR022, Billerica, MA, USA). These cells are ready-to-use primary NSCs isolated from the hippocampus of adult Fisher 344 rats. They were grown in a NSCs expansion medium containing Dulbecco's modified Eagles's medium (DMEM)/F12 medium (Gibco/Invitrogen, Carlsbad, CA) with Lglutamine, B27 supplement, 1× solution of penicillin, streptomycin and fungizone, and basic fibroblast growth factor (bFGF, $20 \mathrm{ng} / \mathrm{mL}$ ). Tissue culture plastic- or glass wares that were used to culture hippocampal NSCs were coated with poly-L-ornithine $(10 \mu \mathrm{g} / \mathrm{mL})$ and laminin $(5$ $\mu \mathrm{g} / \mathrm{mL})$. The hippocampal NSCs were maintained at $37^{\circ} \mathrm{C}$ in a $5 \% \mathrm{CO}_{2}$ humidified incubator and passaged once every 3-4 days. To determine whether ghrelin stimulates the proliferation of hippocampal NSCs, cells were treated with ghrelin $(100 \mathrm{nM})$ for $24 \mathrm{~h}$. All experiments were performed three times in duplicate.

\section{Oxygen-Glucose Deprivation (OGD)}

To induce ischemia, cells were exposed to OGD as previously described, with some modifications [5]. On the day of the experiment, the DMEM/F12 medium was replaced with OGD medium (glucose-free DMEM). Cultures were then placed in a humidified $37^{\circ} \mathrm{C}$ incubator within the Hypoxic Workstation (Daiki Sciences Co. Ltd. by Ruskinn Technology, Bridgend Mid Glamorgan, UK) containing a gas mixture of $1 \% \mathrm{O}_{2}, 5 \% \mathrm{CO}_{2}$, and $94 \% \mathrm{~N}_{2}$ for $3 \mathrm{~h}$ to initiate the ischemic insult. OGD was terminated by replacing the OGD medium with DMEM/F12 medium containing $4.5 \mathrm{mg} / \mathrm{mL}$ glucose, and cultures were incubated for an additional $24 \mathrm{~h}$ under normoxic conditions.

\section{Evaluation of cell proliferation using Cell Counting Kit-8 (CCK-8)}

Experiments for cell viability were performed in 24well plates. Cells were seeded at a density of $1 \times 10^{5}$ cells/mL of DMEM/F12 medium containing bFGF. After $24 \mathrm{~h}$, the media were replaced with fresh media with ghrelin $(100 \mathrm{nM})$ or a vehicle and incubated for $24 \mathrm{~h}$. The cell viability was determined using the cck-8 method (Enzo life science Icn. Lausen, Switzerland) according to the manufacturer's instructions. In brief, $20 \mu \mathrm{L}$ of cck-8 solution was add into each well (containing $200 \mu \mathrm{L}$ of medium), and further cultured for $2 \mathrm{~h}$ at $37^{\circ} \mathrm{C}$. The absorbance of each group at $450 \mathrm{~nm}$ was detected $(n=3)$ using an absorbance microplate reader (Molecular devices LLC., CA, USA) and it was directly proportional to the number of living cells. All experiments were performed three times in triplicate.

\section{Cyto-ID ${ }^{\circledR}$ Green autophagy dye}

To stain cells with Cyto-ID ${ }^{\circledR}$ (Enzo life science Icn. NY, USA) we followed the manufacturer's protocol with modifications. In brief, cells were first washed with PBS (supplemented with $0.2 \%$ B27) and then mixed with either Cyto-ID ${ }^{\circledR}$ solution. The cells were then incubated at $37^{\circ} \mathrm{C}$ for $30 \mathrm{~min}$ in the dark, then washed twice with PBS to remove the free dyes. Cells were fixed in $4 \%$ paraformaldehyde for $20 \mathrm{~min}$ at room temperature then cells were washed twice with PBS, followed by mounting 
with fluorescence mounting media (Dako, CA, USA). Images were acquired by the Carl Zeiss LSM 700 Meta confocal microscope and analyzed using Carl Zeiss ZEN image software. Stained samples were analyzed by flow cytometric analysis using FACSCalibur ${ }^{\mathrm{TM}}$ (Becton Dickinson Biosciences, CA, USA). All experiments were performed three times in duplicate.

\section{Transmission Electron Microscopy}

Cells were fixed in $1 \%$ glutaraldehyde in $0.1 \mathrm{M}$ cacodylate buffer ( $\mathrm{pH} 7)$. Fixed monolayers were scraped; post fixed in $2 \%$ osmium tetroxide, $100 \mathrm{mM}$ cacodylate buffer; dehydrated with increasing concentrations of ethanol; and gradually infiltrated with Epon resin (Pelco, CA, USA). Thin sections were stained with uranyl acetate and lead citrate and examined using the Olympus EM 900 transmission electron microscope.

\section{Transient transfection and identification of autophagy}

GFP-tagged LC3 expression vector was utilized to demonstrate the occurrence of autophagy. Adult rat hippocampal NPCs were seeded $\left(9 \times 10^{3}\right.$ cells/well $)$ in 96-well plates and cultured overnight, then GFP-LC3 expression plasmids were transiently transfected into the cells using Lipofectamin 2000 transfection reagent (Invitrogen, CA, USA), according to the manufacturer's instructions. The cells were subjected to the indicated treatments $24 \mathrm{~h}$ after transfection. At the end of the treatments, cells were fixed in $4 \%$ paraformaldehyde for 20 min at room temperature then cells were washed twice with PBS, followed by mounting with fluorescence mounting media (Dako, CA, USA). The puncta were observed under the Carl Zeiss LSM 700 Meta confocal microscope and analyzed using Carl Zeiss ZEN image software. A minimum of 100 cells per sample was counted in triplicate for each experiment.

\section{Fluorescence-activated cell sorting (FACS) analysis}

Cell viability was examined by FACS analysis. $1 \times 10^{6}$ cells were collected and fixed with 3.7\% PFA. After fixation, the propidium iodide (PI, $50 \mu \mathrm{g} / \mathrm{mL}$ )-staining solution with RNase A (BD Biosciences, CA, USA) was added. After 30 min of incubation, and then the cells were filtered on a nylon mesh filter. Stained samples were analyzed by flow cytometric analysis using FACSCalibur $^{\mathrm{TM}}$ (Becton Dickinson Biosciences, CA, USA). All experiments were performed three times in duplicate.

\section{Western blot analysis}

Cells were lysed in a buffer containing $20 \mathrm{mM}$ Tris$\mathrm{HCl}$ (pH 7.4), 1 mM EDTA, $140 \mathrm{mM} \mathrm{NaCl}, 1 \%$ (w/v) Nonidet P-40, $1 \mathrm{mM} \mathrm{Na} \mathrm{VO}_{4}, 1 \mathrm{mM}$ phenylmethylsulfonyl fluoride, $50 \mathrm{mM} \mathrm{NaF}$, and $10 \mu \mathrm{g} / \mathrm{mL}$ aprotinin. Cell lysates were separated by $12 \%$ SDS-PAGE and electrotransfered onto polyvinylidene difluoride membranes (Bio-Rad, CA, USA). The membranes were soaked in a blocking buffer ( $1 \times$ Tris-buffered saline, $1 \%$ $\mathrm{BSA}$, and $1 \%$ nonfat dry milk) for $1 \mathrm{~h}$ and incubated overnight at $4{ }^{\circ} \mathrm{C}$ with the primary antibodies against LC3-II, Beclin-1 (Novus Biologicals, Littleton, USA; 1:500) and p-62 (MBL, Nagoya, Japan; 1:500) and Bcl-2 (Cell Signaling, Danvers, MA, USA; 1:1000) and $\beta$-actin (Santa Cruz Biotechnology, CA, USA). Blots were developed using a peroxidase-conjugated anti-rabbit IgG and a chemiluminescent detection system (Santa Cruz Biotechnology, CA, USA). The bands were visualized using a ChemicDoc XRS system (Bio-Rad) and quantified using Quantity One imaging software (Bio-Rad, CA, USA). All experiments were performed three times in duplicate.

\section{Statistical analysis}

Data are presented as mean \pm s.e.m. of three different experiments (each experiment was performed in duplicate). Statistical analysis between groups was performed using one-way ANOVA and the Holm-Sidak method for multiple comparisons using SigmaStat for Windows Version 3.10 (Systat Software, Inc., Point Richmond, CA, USA). $p<0.05$ was considered statistically significant.

\section{Results}

\section{Ghrelin protects adult rat hippocampal NSCs against OGD insult}

In previous papers, we reported the protective effect of ghrelin on oxygen-glucose deprivation induced apoptosis [5] and tunicamycin-induced endoplasmic reticulum (ER) stress in neuronal cells [20]. In this study we investigated the effect of ghrelin on hypoxia induced autophagic cell death. After exposure to OGD $1 \% \mathrm{O}_{2}, 5 \% \mathrm{CO}_{2}$, and $94 \% \mathrm{~N}_{2}$ for 3 hours, followed by an additional $24 \mathrm{~h}$ under normoxic conditions then cell viability of OGD group was significantly reduced when compared with the normoxic group (Fig. 1A). Cells are stained with PI and analyzed with a flow cytometer (Fig. 1 B). The percentage of viable cells compared with normoxic controls decreased to $75.7 \%$ (OGD) and was significantly 


\section{A. CCK-8 assay}

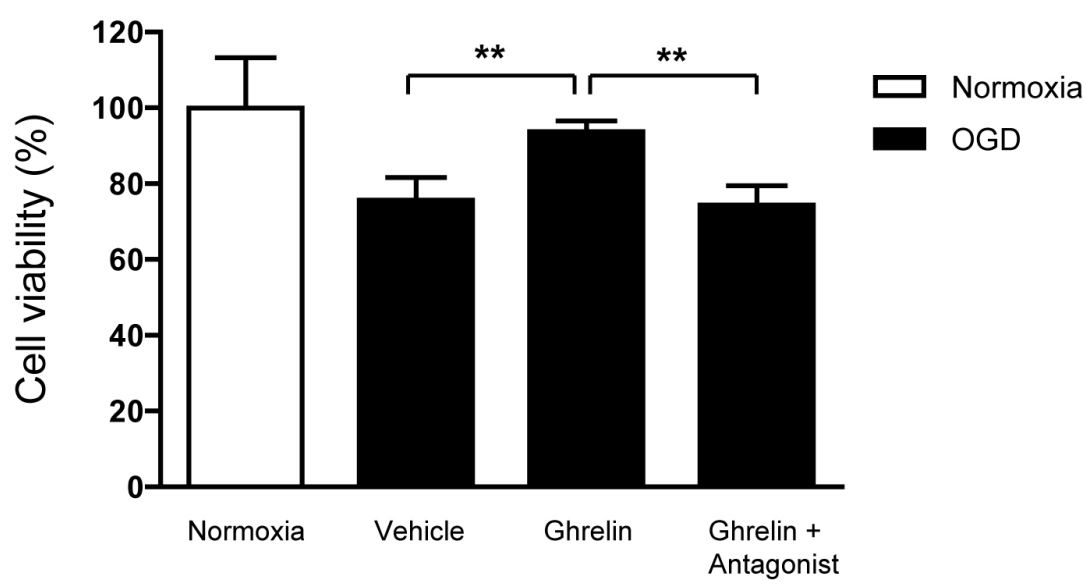

\section{B. PI staining}

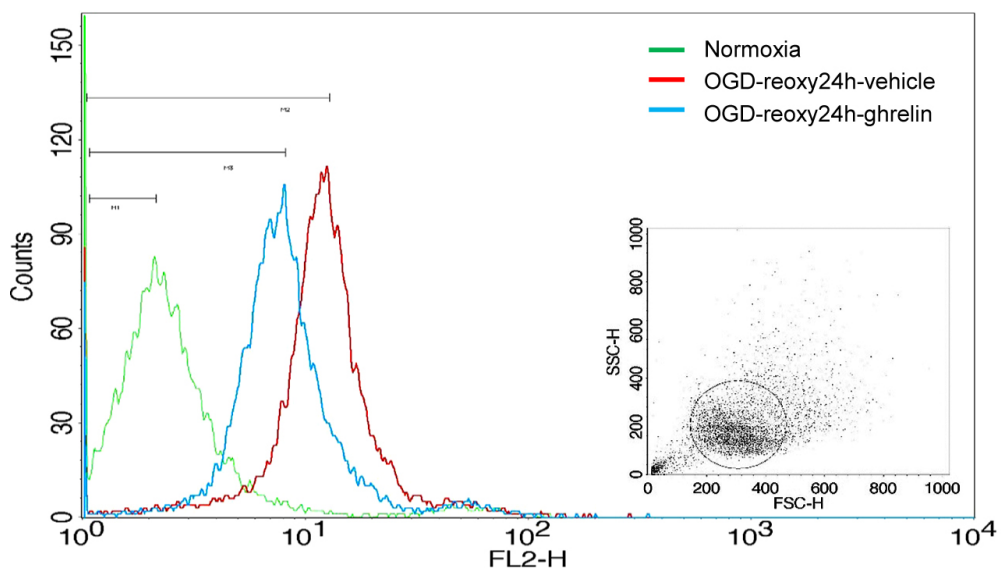

Fig. 1 Ghrelin protects adult rat hippocampal NSCs against OGD insult

Cells were then placed in a humidified $37^{\circ} \mathrm{C}$ incubator within the Hypoxic Workstation containing a gas mixture of $1 \% \mathrm{O}_{2}, 5 \%$ $\mathrm{CO}_{2}$, and $94 \% \mathrm{~N}_{2}$ for $3 \mathrm{~h}$ to initiate the ischemic insult. OGD was terminated by replacing the OGD medium with DMEM/F12 medium containing $4.5 \mathrm{mg} / \mathrm{mL}$ glucose, and cells were incubated for an additional $24 \mathrm{~h}$ under normoxic conditions. (A) OGD insulted cells were treated with ghrelin $(100 \mathrm{nM})$ for $27 \mathrm{~h}$ and the CCK-8 methods was used to analyze the viability of cells. (B) OGD insulted cells were harvested by stained with propidium iodide (PI, $50 \mu \mathrm{g} / \mathrm{mL}$ )-staining solution with RNase A (BD Biosciences). Results were expressed graphically and quantified as the fraction of cells in normoxia, OGD insult treated vehicle or ghrelin using CellQuest software (Beckman Coulter). The data are expressed as the mean \pm S.E.M. of three different experiments (each experiment was performed in duplicate). ${ }^{* *} p<0.01 v s$. the Normoxia or OGD- vehicle.

increased to $93.7 \%$ ( $p<0.05$ vs. OGD-vehicle control) by pretreatment with ghrelin. It has been reported that the adult rat hippocampal NSCs express GHS-R1a [21]. The exposure of cells to the receptor-specific antagonist D-Lys-3-GHRP-6 $\left(10^{-6} \mathrm{M}\right)$ abolished the protective effect of ghrelin against OGD insult. Cell viability assessed by cell counting kit- 8 assay. We found that ghrelin treated cells showed protective effect against OGD-induced cell death.

\section{OGD strongly induces autophagy markers in adult rat hippocampal NSCs}

To further determine the OGD induced autophagy activity in the adult rat hippocampal NSCs, we examined the autophagy activity using Cyto-ID analysis. OGD strongly induces autophagy activity in adult rat hippocampal NSCs (Fig. 2A). It has been reported that hypoxic injury triggered the expression of a number of markers for autophagy markers, such as microtubule- 


\section{A. FACS analysis}

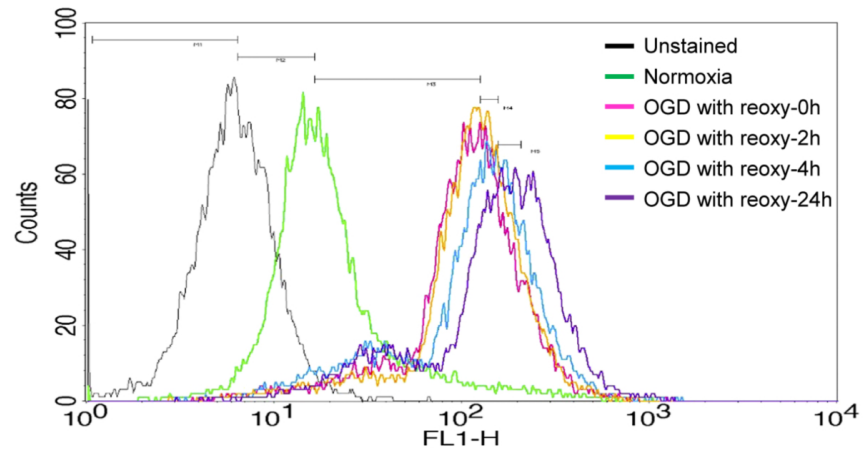

\section{B. Western blotting}
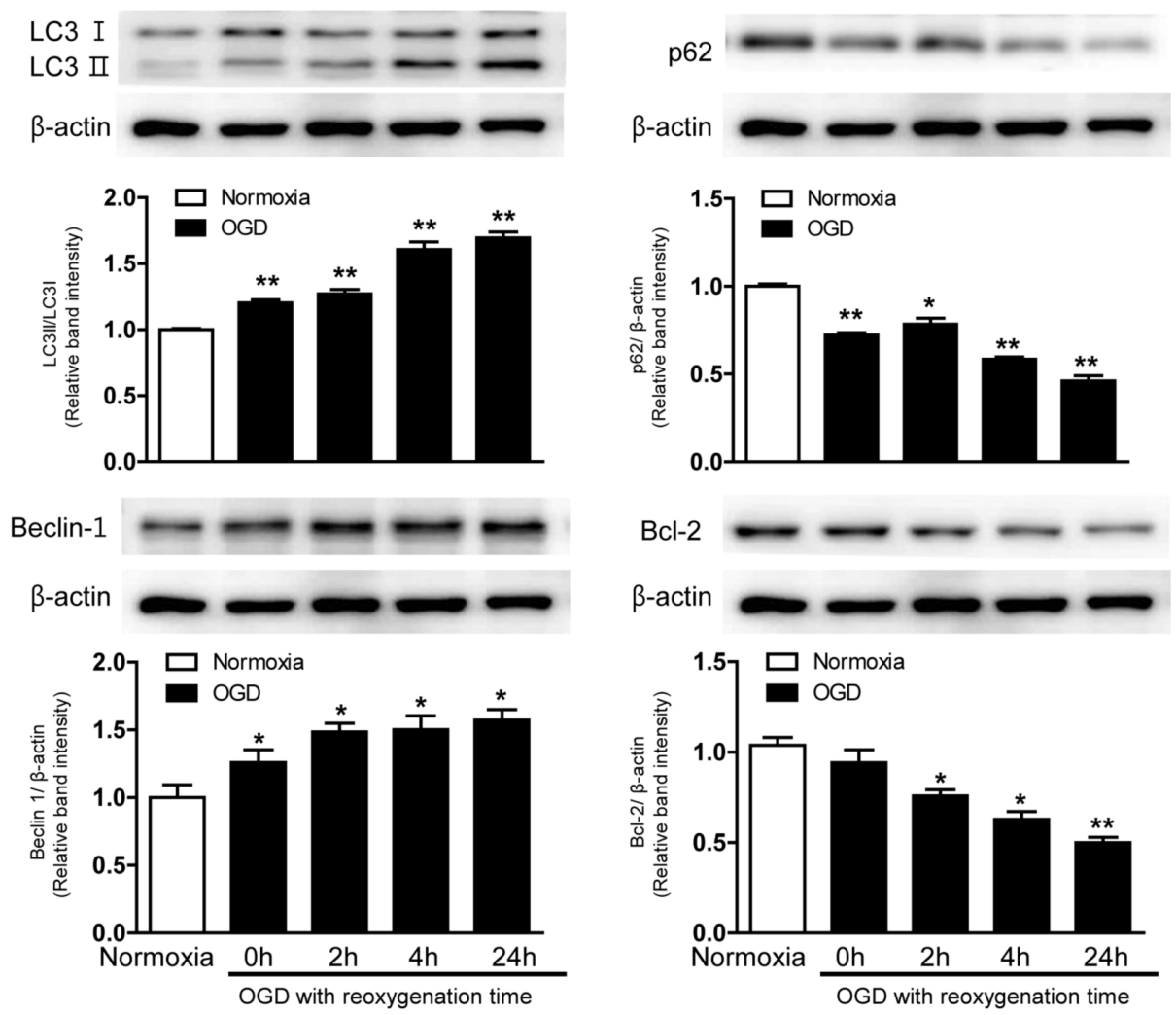

Fig. 2 OGD strongly induces autophagy markers in adult rat hippocampal NSCs

Cells were then placed in a humidified $37^{\circ} \mathrm{C}$ incubator within the Hypoxic Workstation containing a gas mixture of $1 \% \mathrm{O}_{2}, 5 \%$ $\mathrm{CO}_{2}$, and $94 \% \mathrm{~N}_{2}$ for $3 \mathrm{~h}$ to initiate the ischemic insult. OGD was terminated by replacing the OGD medium with DMEM/F12 medium containing $4.5 \mathrm{mg} / \mathrm{mL}$ glucose, and cells were incubated for an additional 0, 2, 4, $24 \mathrm{~h}$ under normoxic conditions. (A) To further determine the OGD induced autophagy activity in the adult rat hippocampal NSCs, we examined the autophagy activity using Cyto-ID ${ }^{\circledR}$ assay. The cells were then incubated at $37^{\circ} \mathrm{C}$ for $30 \mathrm{~min}$ in the dark, then washed twice with PBS to remove the free dyes. Cells were fixed in 4\% paraformaldehyde for $20 \mathrm{~min}$ at room temperature then cells were washed twice with PBS, followed by mounting with fluorescence mounting media. Stained samples were analyzed by flow cytometric analysis using FACSCalibur $^{\mathrm{TM}}$. All experiments were performed three times in duplicate. (B) Protein lysates were prepared and assessed by western blot analysis using anti-LC3-II, anti- Beclin-1, anti-p62, anti-Bcl-2 and anti- $\beta$-actin antibodies. The band intensities of LC3-II, Beclin-1, p62, Bcl-2 were normalized to the band intensities of $\beta$-actin and they are expressed as relative band intensities. The results are representative of three independent experiments. The data are expressed as the mean \pm S.E.M. of three different experiments (each experiment was performed in duplicate). ${ }^{*} p<0.05,{ }^{* *} p<0.01 v s$. the Normoxia. 
associated protein 1 light chain 3B (LC II), Beclin-1 and p62 in neuronal cells. As shown in Fig. 2B, OGD induced the expression of these markers in the adult rat hippocampal NSCs. LC3-II (16 KD) level was remarkably increased, while LC3-I (18 KD) level unchanged under the hypoxic conditions. The ratio of LC3-II/LC3-I was also increased in an OGD insult time dependent manner. The expression levels of $\mathrm{p} 62$ protein, a well-known autophagic substrate, were significantly decreased in the OGD-insulted cells. One of the key mechanisms for control of autophagy is the modulation of the autophagic protein Beclin-1 and the anti-apoptotic Bcl-2 protein expression [22]. In addition, Bcl-2 level was decreased but Beclin-1 level was significantly increased under the hypoxic conditions.

\section{Ghrelin inhibits OGD induced excessive autophagy markers}

LC3 is correlated with the autophagosomal membranes that exist in two molecular forms: LC3-I (18 kDa) and LC3-II (16 kDa). The cytoplasmic LC3-I form is converted into the LC3-II form, which is recruited to autophagosomal membranes during autophagy activation. Beclin-1 is one component of a protein complex with PI3K, which has an important role in membrane trafficking and restructuring involved in the formation of autophagosomes [23]. Ghrelin significantly attenuated OGD induced LC3-II (Fig. 3A) and Beclin-1 (Fig. 3B) protein level upregulation. Also, ghrelin treated cells showed an increased OGD induced p62 (Fig. 3C) and Bcl-2 (Fig. 3D) protein level downregulation.

\section{Ghrelin suppresses OGD induced excessive autophagy activity}

Cyto-ID ${ }^{\circledR}$ assay is based on the usage of a specific dye that Flow Cytometric Analysis or fluorescence microscopy of autophagic activity with Cyto-ID ${ }^{\circledR}$ Green autophagy dye staining in cells. Here, we investigated the effect of ghrelin on OGD induced autophagy activity in adult rat hippocampal NPCs as determined by flow cytometric analysis or confocal microscope. Ghrelin suppressed OGD induced autophagy activity using flow cytometric analysis (Fig. 4A). In addition, ghrelin treated cells showed a dramatically decreased green fluorescence dye using Carl Zeiss LSM 700 Meta confocal microscope (Fig. 4B).

\section{Ghrelin reduces autophagosome formation and number of GFP-LC3 transfected puncta}

Transmitted electron microscopy (TEM) also showed that vacuolar structures containing cytoplasm were clearly encircled by double membrane structures, resembling autophagosomes and autophagolysosome, in OGDinsulted adult rat hippocampal NSCs (Fig. 5A). There was rare or no autophagosome or autophagolysosome in the control. Ghrelin significantly attenuated OGD induced autophagosomes and autophagolysosome formation in adult rat hippocampal NSCs. In addition, ghrelin remarkably reduced number of GFP-LC3 transfected puncta (Fig. 5B).

\section{Discussion}

In the present study, we demonstrated that ghrelin protects adult hippocampal NSCs from the OGD-induced excessive autophagy cell death via the inhibition of autophagy activity markers and suppression of autophagosomes and autophagolysosome formation. The neuroprotective effect of ghrelin be associated with the inhibition of OGD-induced autophagy activity and the suppression of autophagy marker, such as LC3-II, Beclin-1 and activation of $\mathrm{p} 62$ and $\mathrm{Bcl}-2$ during OGD stress responses. In this study, we showed for the first time that ghrelin rescues adult rat hippocampal NSCs from OGD stress-induced excessive autophagy cell death.

Ghrelin is a newly discovered peptide with various functions, including regulation energy metabolism[24], food intake and glucose homeostasis [25], promoting cell proliferation. In addition, ghrelin has been recently been shown to have, including CNS to control neuronal function[26] and various brain injury [27, 28]. Several growth factors, including bFGF and insulin like growth factor have been implicated in neuroprotective effect. The major finding of this study is that the potent neuroprotective agent ghrelin protects the OGD induced cell death of adult rat hippocampal NSCs via regulating autophagic activity.

Autophagy is activated by nutrient starvation and growth factor deprivation when cells are unable to take up external nutrients. In this study, autophagy was observed within 4 hours under hypoxic condition, and the number of autophagosomes further increased during the reperfusion phase $(0,2,4,24 \mathrm{~h})$. LC3-II and Beclin-1 are essential components in the process of autophagy. Beclin-1 and LC3-II were reported to be increased during reperfusion in fibrillated mouse hearts [29]. And 
A

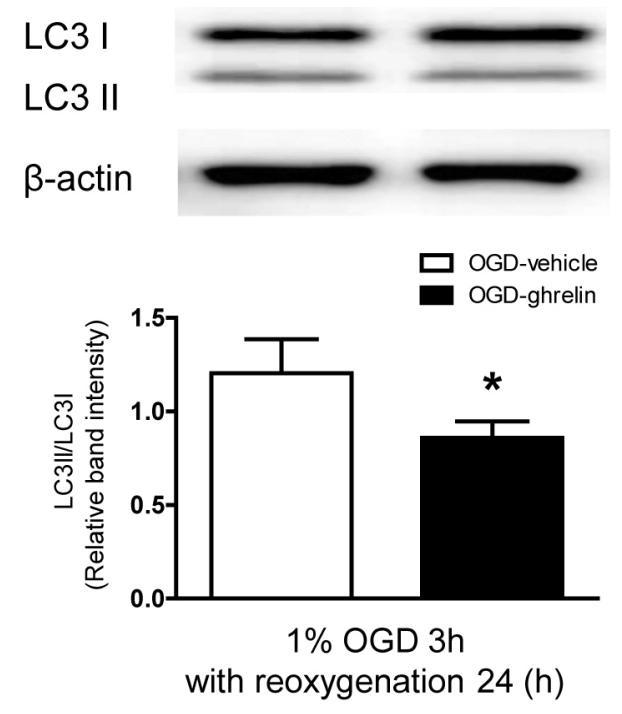

C

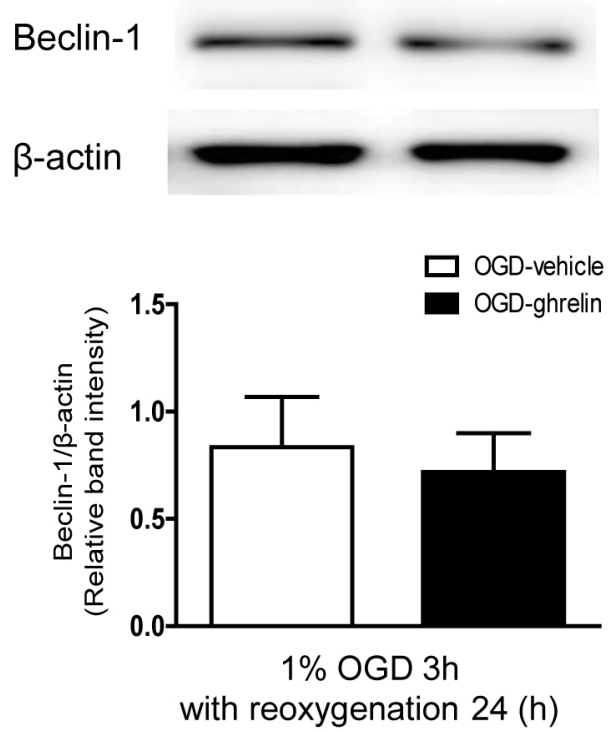

$\mathbf{B}$

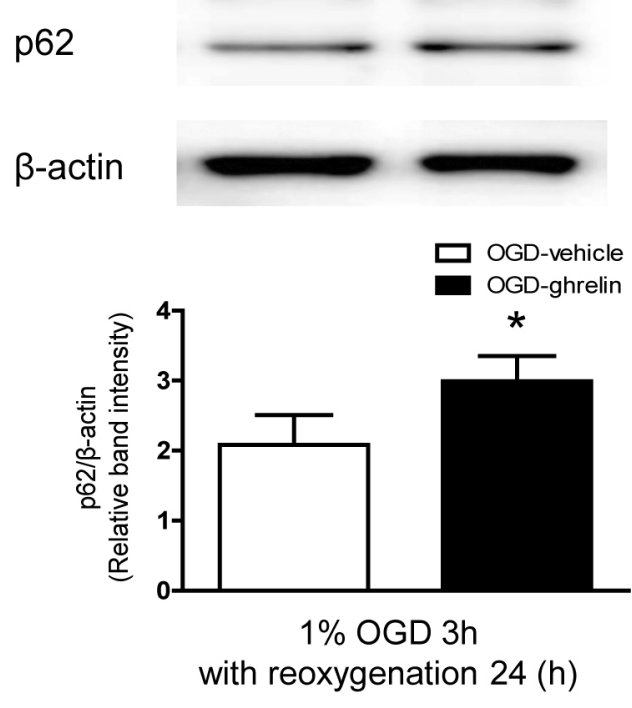

D

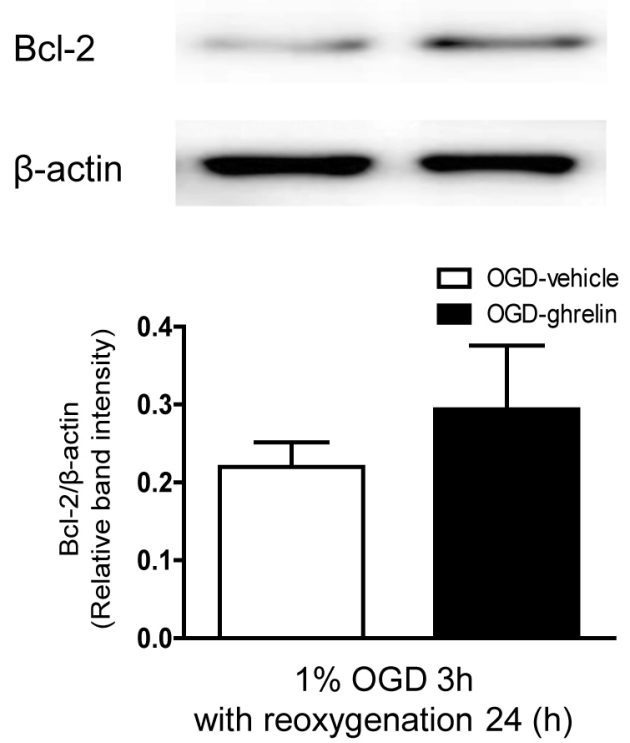

Fig. 3 Ghrelin inhibits OGD induced excessive autophagy markers

OGD $\left(1 \% \mathrm{O}_{2}, 5 \% \mathrm{CO}_{2}\right.$, and $94 \% \mathrm{~N}_{2}$ for $\left.3 \mathrm{~h}\right)$ was terminated by replacing the OGD medium with DMEM/F12 medium containing $4.5 \mathrm{mg} / \mathrm{mL}$ glucose, and cells were incubated for an additional $24 \mathrm{~h}$ under normoxic conditions. OGD insulted cells were treated with ghrelin $(100 \mathrm{nM})$ for $27 \mathrm{~h}$. Protein lysates were prepared and assessed by western blot analysis using anti- LC3-II, antiBeclin-1, anti-p62, anti-Bcl-2 and anti-g $\beta$-actin antibodies. The band intensities of LC3-II, Beclin-1, p62, Bcl-2 were normalized to the band intensities of $\beta$-actin and they are expressed as relative band intensities. The results are representative of three independent experiments.

recently reported to OGD/reoxygenation-induced increased expression of Beclin-1 and LC3-II in hippocampal neurons [30]. LC3, the rat microtubule-associated protein $1 \mathrm{~A}$ light chain 3 , is normally located throughout the cytoplasm but becomes concentrated in the mem- branes of autophagosomes after cleavage of a few amino acid at the C-terminus (LC3-II) during maturation of autophagy [31]. Beclin-1 was first described as a Bcl-2interacting protein and was formerly found to promote autophagy [32]. Beclin-1 was expressed in many areas 


\section{A. FACS analysis}

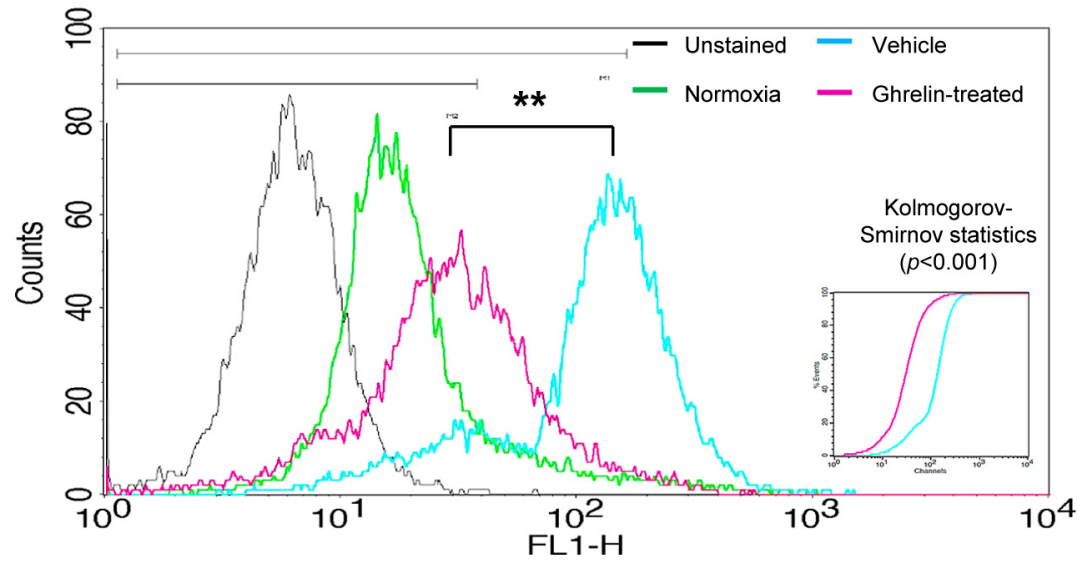

B. Confocal image

OGD ( $1 \%$ O2 $3 \mathrm{~h}$ with $24 \mathrm{~h}$ reoxygenation )
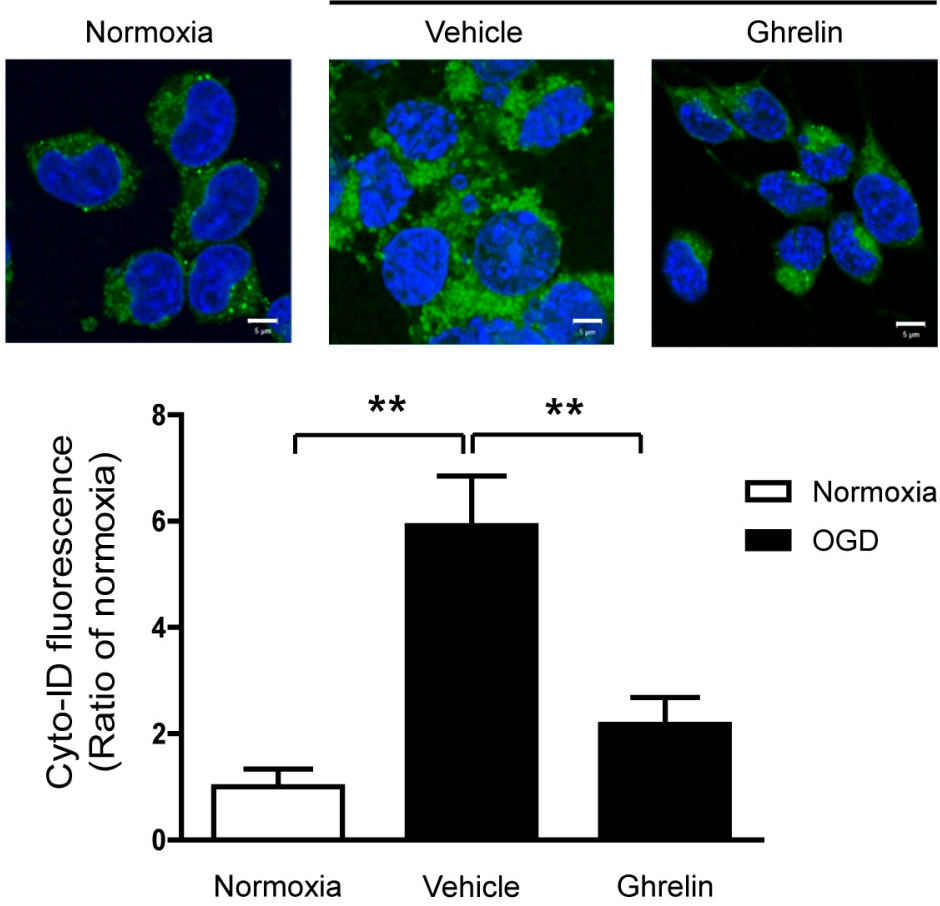

Fig. 4 Ghrelin suppresses OGD induced excessive autophagy activity

OGD $\left(1 \% \mathrm{O}_{2}, 5 \% \mathrm{CO}_{2}\right.$, and $94 \% \mathrm{~N}_{2}$ for $\left.3 \mathrm{~h}\right)$ was terminated by replacing the OGD medium with DMEM/F12 medium containing $4.5 \mathrm{mg} / \mathrm{mL}$ glucose, and cells were incubated for an additional $24 \mathrm{~h}$ under normoxic conditions. OGD insulted cells were treated with ghrelin $(100 \mathrm{nM})$ for $27 \mathrm{~h}$. To further determine the OGD induced autophagy activity in the adult rat hippocampal NSCs, we examined the autophagy activity using Cyto-ID ${ }^{\circledR}$ assay. (A) Stained samples were analyzed by flow cytometric analysis using FACSCalibur $^{\mathrm{TM}}$. (B) Stained samples were analyzed by Carl Zeiss LSM 700 Meta confocal microscope. The results are representative of three independent experiments.

including cerebral cortex, hippocampus, and cerebellum in the rat brain [33]. As expected, we found that after OGD, LC3-II and Beclin-1 expression in adult rat hippo- campal NPCs were up regulated.

It is well-known that ghrelin is mainly produced by endocrine cells in the gastric oxyntic mucosa [1]. Given 


\section{A. EM (Electron microscopy)}

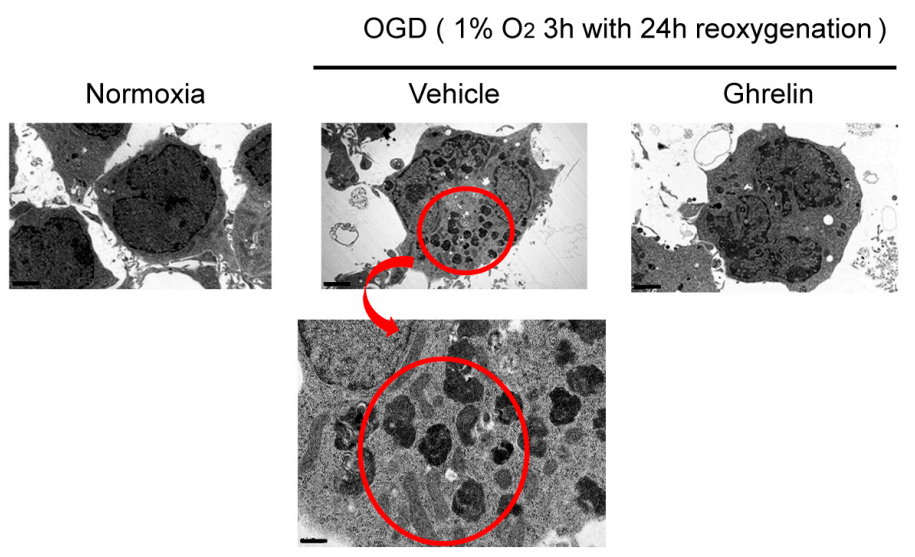

\section{B. GFP-LC3 puncta assay}

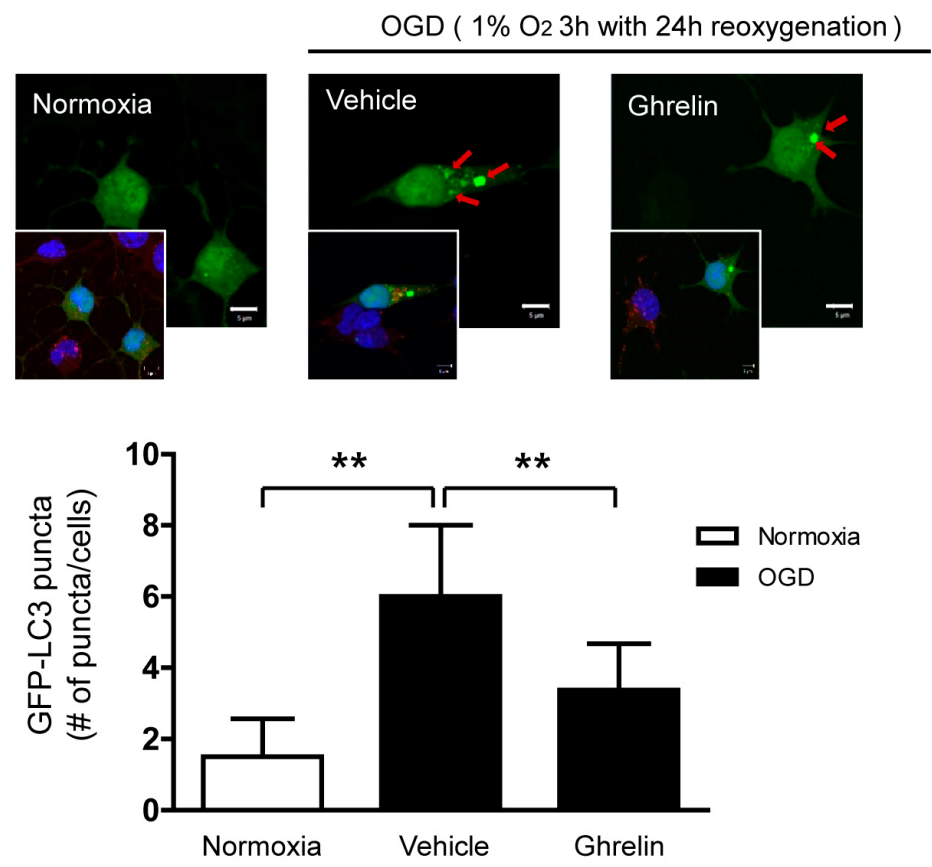

Fig. 5 Ghrelin reduces autophagosome formation and number of GFP-LC3 transfected puncta

OGD $\left(1 \% \mathrm{O}_{2}, 5 \% \mathrm{CO}_{2}\right.$, and $94 \% \mathrm{~N}_{2}$ for $\left.3 \mathrm{~h}\right)$ was terminated by replacing the OGD medium with DMEM/F12 medium containing $4.5 \mathrm{mg} / \mathrm{mL}$ glucose, and cells were incubated for an additional $24 \mathrm{~h}$ under normoxic conditions. OGD insulted cells were treated with ghrelin (100 nM) for $27 \mathrm{~h}$. (A) Cells were fixed in $1 \%$ glutaraldehyde in $0.1 \mathrm{M}$ cacodylate buffer (pH 7). Fixed monolayers were scraped; post fixed in $2 \%$ osmium tetroxide, $100 \mathrm{mM}$ cacodylate buffer; dehydrated with increasing concentrations of ethanol; and gradually infiltrated with Epon resin (Pelco). Thin sections were stained with uranyl acetate and lead citrate and examined using the Olympus EM 900 transmission electron microscope. Accumulation of GFP-LC3 transfected puncta occurs upon OGD induced adult hippocampal NPCs. (B) Cells were transfected with GFP-LC3 and after $24 \mathrm{~h}$ cells were incubated under hypoxic conditions. At the end of the treatments, cells were fixed in 4\% paraformaldehyde for 20 min at room temperature then cells were washed twice with PBS, followed by mounting with fluorescence mounting media. The puncta were observed under the Carl Zeiss LSM 700 Meta confocal microscope and analyzed using Carl Zeiss ZEN image software. A minimum of 100 cells per sample was counted in triplicate for each experiment. The data are expressed as the mean \pm S.E.M. of three different experiments (each experiment was performed in duplicate). ${ }^{*} p<0.05$ vs. the OGD-vehicle. 
the fact that ghrelin can access the brain via diffusion [34] and the serum ghrelin levels were increased in middle cerebral artery occluded rats [35], these findings suggest that peripherally synthesized ghrelin may contribute to the protection of adult hippocampal NSCs during OGD. Moreover, plasma ghrelin levels are elevated [36] and survival of newborn cells in the dentate gyrus of the hippocampus is increased [37] in calorie-restricted animals. In contrast, there is still much room for debate if ghrelin is synthesized in the brain because the evidences of central production of ghrelin have been inconsistent. Earlier immunocytochemical studies suggested the presence of ghrelin in some brain areas, such as the hypothalamus [38] and the cortex [39]. However, it has been reported that no ghrelin-specific staining is found and ghrelin receptor-expressing neurons do not have adjacent ghrelin immunoreactive terminals in the brain or spinal cord of the rat and mouse [40]. Recently, Cabral et al. [41] provided a comprehensive review of all available data related to the distribution of ghrelin in the CNS and concluded that there are no indisputable and reliable evidences to support the idea that ghrelin is present in the brain at physiologically significant levels.

In conclusion, our data demonstrated that ghrelin pro- tected against OGD induced autophagy cell death which regulated the excessive autophagy in adult rat hippocampal NSCs. Furthermore, the OGD induced excessive autophagy activity downregulation may be responsible for the neuroprotective agent. Taken together, our results for the first time suggested that the protective effect of ghrelin under hypoxic condition was regulated by autophagy. Regulating autophagic activity may be a potential optimizing target for promoting adult hippocampal NSCs based therapy for stroke.

\section{Declaration of Interest}

The authors declare that there is no conflict of interest that could be perceived as prejudicing the impartiality of the research reported.

\section{Funding}

This research was supported by the National Research Foundation of Korea (NRF) grant funded by the Korea government (MSIP) (No. 2011-0030072) (to S.P.) and (No. 2014R1A1A2058316) (to H.C.)

\section{References}

1. Kojima M, Hosoda H, Date Y, Nakazato M, Matsuo H, et al. (1999) Ghrelin is a growth-hormone-releasing acylated peptide from stomach. Nature 402: 656-660.

2. Hosoda H, Kojima M, Matsuo H, Kangawa K (2000) Ghrelin and des-acyl ghrelin: two major forms of rat ghrelin peptide in gastrointestinal tissue. Biochem Biophys Res Commun 279: 909-913.

3. Broglio F, Benso A, Gottero C, Prodam F, Gauna C, et al. (2003) Non-acylated ghrelin does not possess the pituitaric and pancreatic endocrine activity of acylated ghrelin in humans. J Endocrinol Invest 26: 192-196.

4. Cruz SA, Tseng YC, Kaiya H, Hwang PP (2010) Ghrelin affects carbohydrate-glycogen metabolism via insulin inhibition and glucagon stimulation in the zebrafish (Danio rerio) brain. Comp Biochem Physiol A Mol Integr Physiol 156: 190-200.

5. Chung H, Kim E, Lee DH, Seo S, Ju S et al. (2007) Ghrelin inhibits apoptosis in hypothalamic neuronal cells during oxygen-glucose deprivation. Endocrinology 148: 148-159.

6. Chung H, Seo S, Moon M, Park S (2008) Phosphatidylinositol3-kinase/Akt/glycogen synthase kinase-3 beta and ERK1/2 pathways mediate protective effects of acylated and unacylated ghrelin against oxygen-glucose deprivation-induced apoptosis in primary rat cortical neuronal cells. J Endocrinol 198: 511521.

7. Moon M, Kim HG, Hwang L, Seo JH, Kim S, et al. (2009) Neuroprotective effect of ghrelin in the 1methyl-4-phenyl-1,2,3,6-tetrahydropyridine mouse model of Parkinson's disease by blocking microglial activation. Neurotox Res 15: 332-347.

8. Hwang S, Moon M, Kim S, Hwang L, Ahn KJ, et al. (2009) Neuroprotective effect of ghrelin is associated with decreased expression of prostate apoptosis response-4. Endocr J 56: 609-617.

9. Lee JY, Chung H, Yoo YS, Oh YJ, Oh TH, et al. (2010) Inhibition of apoptotic cell death by ghrelin improves functional recovery after spinal cord injury. Endocrinology 151: 3815-3826.

10. Ersahin M, Toklu HZ, Erzik C, Cetinel S, Akakin D, et al. (2010) The anti-inflammatory and neuroprotective effects of ghrelin in subarachnoid hemorrhage-induced oxidative brain damage in rats. J Neurotrauma 27: 1143-1155.

11. Jang W, Kim HJ, Li H, Jo KD, Lee MK, et al. (2016) The Neuroprotective Effect of Erythropoietin on RotenoneInduced Neurotoxicity in SH-SY5Y Cells Through the 
Induction of Autophagy. Mol Neurobiol 53: 3812-3821.

12. Xia DY, Li W, Qian HR, Yao S, Liu JG, et al. (2013) Ischemia preconditioning is neuroprotective in a rat cerebral ischemic injury model through autophagy activation and apoptosis inhibition. Braz J Med Biol Res 46: 580588.

13. Kim HJ, Kim J, Kang KS, Lee KT, Yang HO (2014) Neuroprotective effect of chebulagic acid via autophagy induction in SH-SY5Y cells. Biomol Ther (Seoul) 22: 275-281.

14. Wong E, Cuervo AM (2010) Autophagy gone awry in neurodegenerative diseases. Nat Neurosci 13: 805-811.

15. Shi R, Weng J, Zhao L, Li XM, Gao TM, et al. (2012) Excessive autophagy contributes to neuron death in cerebral ischemia. CNS Neurosci Ther 18: 250-260.

16. Huang Z, Han Z, Ye B, Dai Z, Shan P, et al. (2015) Berberine alleviates cardiac ischemia/reperfusion injury by inhibiting excessive autophagy in cardiomyocytes. Eur J Pharmacol 762: 1-10.

17. Lipton P (1999) Ischemic cell death in brain neurons. Physiol Rev 79: 1431-1568.

18. Descloux C, Ginet V, Clarke PG, Puyal J, Truttmann AC (2015) Neuronal death after perinatal cerebral hypoxiaischemia: focus on autophagy-mediated cell death. Int $J$ Dev Neurosci 45: 75-85.

19. Chu CT (2008) Eaten alive: autophagy and neuronal cell death after hypoxia-ischemia. Am J Pathol 172: 284-287.

20. Chung H, Chung HY, Bae CW, Kim CJ, Park S (2011) Ghrelin suppresses tunicamycin- or thapsigargin-triggered endoplasmic reticulum stress-mediated apoptosis in primary cultured rat cortical neuronal cells. Endocr $J$ 58: 409-420.

21. Chung H, Li E, Kim Y, Kim S, Park S (2013) Multiple signaling pathways mediate ghrelin-induced proliferation of hippocampal neural stem cells. J Endocrinol 218: 49-59.

22. Pattingre S, Tassa A, Qu X, Garuti R, Liang XH, et al. (2005) Bcl-2 antiapoptotic proteins inhibit Beclin 1dependent autophagy. Cell 122: 927-939.

23. Dong W, Xiao S, Cheng M, Ye X, Zheng G (2016) Minocycline induces protective autophagy in vascular endothelial cells exposed to an in vitro model of ischemia/ reperfusion-induced injury. Biomed Rep 4: 173-177.

24. Heppner KM, Muller TD, Tong J, Tschop MH (2012) Ghrelin in the control of energy, lipid, and glucose metabolism. Methods Enzymol 514: 249-260.

25. Siejka A, Ruxer J, Loba J (2005) [Ghrelin-role in energy homeostasis and glucose metabolism]. Endokrynol Diabetol Chor Przemiany Materii Wieku Rozw 11: 181-185. (In Polish)

26. Andrews ZB (2011) The extra-hypothalamic actions of ghrelin on neuronal function. Trends Neurosci 34: 31-40.

27. Sun N, Wang H, Ma L, Lei P, Zhang Q (2016) Ghrelin attenuates brain injury in septic mice via PI3K/Akt signaling activation. Brain Res Bull 124: 278-285.
28. Ku JM, Taher M, Chin KY, Barsby T, Austin V, et al. (2016) Protective actions of des-acylated ghrelin on brain injury and blood-brain barrier disruption after stroke in mice. Clin Sci (Lond) 130: 1545-1558.

29. Meyer G, Czompa A, Reboul C, Csepanyi E, Czegledi A, et al. (2013) The cellular autophagy markers Beclin-1 and LC3B-II are increased during reperfusion in fibrillated mouse hearts. Curr Pharm Des 19: 6912-6918.

30. Yang N, Li L, Li Z, Ni C, Cao Y, et al. (2017) Protective effect of dapsone on cognitive impairment induced by propofol involves hippocampal autophagy. Neurosci Lett 649: 85-92.

31. Kabeya Y, Mizushima N, Ueno T, Yamamoto A, Kirisako $\mathrm{T}$, et al. (2000) LC3, a mammalian homologue of yeast Apg8p, is localized in autophagosome membranes after processing. EMBO J 19: 5720-5728.

32. Gaytan M, Morales C, Sanchez-Criado JE, Gaytan F (2008) Immunolocalization of beclin 1, a bcl-2-binding, autophagy-related protein, in the human ovary: possible relation to life span of corpus luteum. Cell Tissue Res 331: 509-517.

33. Verhaar R, Drukarch B, Bol JG, Jongenelen CA, Wilhelmus MM (2013) Tissue transglutaminase crosslinks beclin 1 and regulates autophagy in MPP(+)-treated human SH-SY5Y cells. Neurochem Int 62: 486-491.

34. Banks WA, Tschop M, Robinson SM, Heiman ML (2002) Extent and direction of ghrelin transport across the bloodbrain barrier is determined by its unique primary structure. J Pharmacol Exp Ther 302: 822-827.

35. Xu X, Zhu Y, Chuai J (2012) Changes in serum ghrelin and small intestinal motility in rats with ischemic stroke. Anat Rec (Hoboken) 295: 307-312.

36. Lutter M, Sakata I, Osborne-Lawrence S, Rovinsky SA, Anderson JG, et al. (2008) The orexigenic hormone ghrelin defends against depressive symptoms of chronic stress. Nat Neurosci 11: 752-753.

37. Lee J, Seroogy KB, Mattson MP (2002) Dietary restriction enhances neurotrophin expression and neurogenesis in the hippocampus of adult mice. J Neurochem 80: 539-547.

38. Lu S, Guan JL, Wang QP, Uehara K, Yamada S, et al. (2002) Immunocytochemical observation of ghrelincontaining neurons in the rat arcuate nucleus. Neurosci Lett 321: 157-160.

39. Hou Z, Miao Y, Gao L, Pan H, Zhu S (2006) Ghrelincontaining neuron in cerebral cortex and hypothalamus linked with the DVC of brainstem in rat. Regul Pept 134: 126-131.

40. Furness JB, Hunne B, Matsuda N, Yin L, Russo D, et al. (2011) Investigation of the presence of ghrelin in the central nervous system of the rat and mouse. Neuroscience 193: 1-9.

41. Cabral A, Lopez Soto EJ, Epelbaum J, Perello M (2017) Is ghrelin synthesized in the central nervous system? Int $J$ Mol Sci 18: 638. 